\title{
Thermoablation in spinal osteoid osteomas
}

\begin{abstract}
Aim: The purpose of this work is to describe our experience in treating osteoid osteomas (OO) of the spine and compare results of surgical resection and percutaneous CT-guided radiofrequency ablation (RFA). Material and

Methods: Retrospective review of 8 cases of OO of the spine: 4 treated with surgical resection and 4 treated with RFA. All had persistent pain after conservative treatment with nonsteroidal anti-inflammatory drugs (NSAID). Results: All patients refer complete relief from nocturnal pain, proving the efficacy of both methods.

Conclusion: Complete resection although historically considered treatment of choice for $\mathrm{OO}$ with very good success rates, can present complications. To minimize surgical damage a precise location of the nidus is crucial. Percutaneous radiofrequency ablation under CT guidance is both efficient and safe being less invasive, performed under local anesthesia and allowing for a faster recovery, lowers costs and shorter hospital stays.

The post-operative complications are similar in both methods. New protective techniques allow thermoablation of OO located near neurological structures with increased confidence and safety.
\end{abstract}

Keywords: Osteoid osteoma, percutaneous radiofrequency ablation, spine, treatment
Volume 10 Issue 3 - 2018

\author{
Pedro Manuel Serrano, Tiago Amorim \\ Barbosa, Ana Ribau, Marta Santos Silva,Vânia \\ Oliveira, Pedro Cardoso \\ Serviço de Ortopedia e Traumatologia, Hospital de Santo \\ António, Portugal
}

\author{
Correspondence: Pedro Manuel Serrano, Serviço de \\ Ortopedia e Traumatologia, Centro Hospitalar do Porto, \\ Hospital de Santo António, Largo Professor Abel Salazar, 4099. \\ 00I Porto, Portugal, Tel +35I 919069834, \\ Emailpms1983@gmail.com
}

Received: June 01, 2018 | Published: June 08, 2018

\section{Introduction}

The Osteoid osteoma (OO) is a painful, self-limited bone tumor that can persist for several weeks, months or even years. Typically, the pain is worse at night and often is controlled with nonsteroidal antiinflammatory drugs (NSAID). ${ }^{1}$ It commonly occurs in male teenagers and young adults, with predilection for long bones of the lower limbs (femur and tibia). ${ }^{2}$ Ten to twenty percent of the lesions occur in the spine, with painful scoliosis and muscle spasm. The posterior elements of the vertebrae are the more affected structures. ${ }^{3-5}$

The diagnosis may be hard, and imaging through CT scan and Bone Scan are essential. The treatment options for the disabling pain and spinal deformity caused by $\mathrm{OO}$ include surgical resection and percutaneous CT-guided radiofrequency ablation (RFA). ${ }^{6,7}$ The exact location of the "nidus" through imaging techniques is very helpful especially when considering it is often hard to identify during surgery. This particular aspect, allied with the benefits of a minimally invasive approach, have been responsible for the increased popularity of RFA. ${ }^{8}$ The proximity to the neurological structures however has been seen by many as a limitation to RFA. With this in mind, new techniques have been described with injection of gas or a refrigerated liquid in the epidural space as a protective barrier between the nidus and the spinal cord. ${ }^{9}$ Several clinical reports of the successful treatment with RFA have been published. ${ }^{10-14}$

The authors report their experience in treating OO of the spine and comment on the use of new techniques to perform RFA near neurological structures.

\section{Case report}

The authors present 8 cases of OO of the spine: 4 treated with surgical resection and 4 treated with RFA. All patients were treated by resection or RFA for persistent pain after conservative treatment with NSAID.

In the group of patients treated by surgical resection, the mean age was 20 years old (16-28), with the osteoid osteoma located at L2 lamina (Figure 1), L2 pediculum (Figure 2), L3 lamina (Figure 3 ) and L4 superior articular process (Figure 4). In the first case, only curettage was performed while in the others, posterior arthrodesis at two levels was also performed. After 4 years of follow-up, all the patients were asymptomatic, with no signs of relapse of the disease.

In RFA group, the mean age was 19 years old (11-28), and the osteoid osteomas were located at the vertebral body of D8 (Figure 5), L2 pediculum (Figure 6), L4 pediculum (Figure 7) and at the second sacral vertebra (Figure 8). In the last case, the patient presented an irradiated pain to the ipsilateral limb that resolved completely after 48 hours. No other complications were found. After 1 year of follow-up, all patients were asymptomatic.

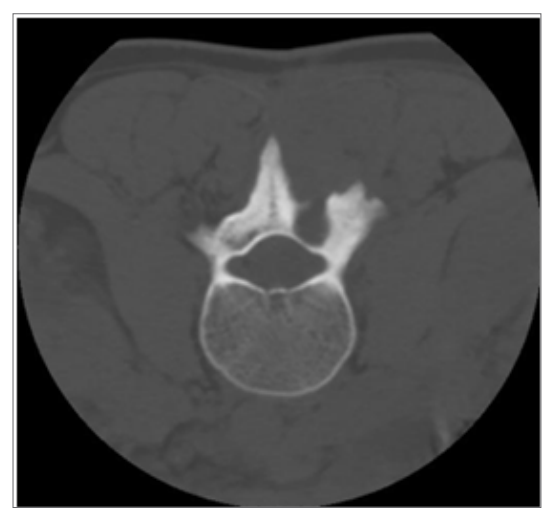

Figure I Axial CT-scan of Osteoid osteoma of L2 left lamina. 

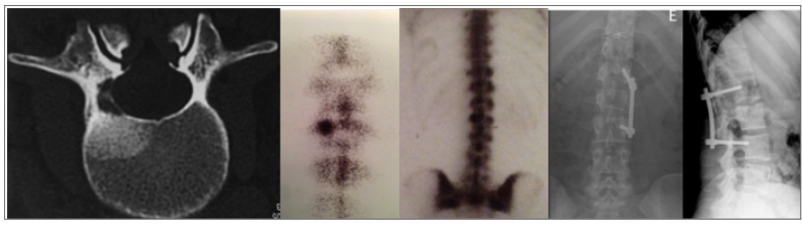

Figure 2 Axial CT-scan and bone scintigraphy of an Osteoid osteoma of L2 right pediculum and plain radiograph after LI-L3 posterior spine instrumentation.

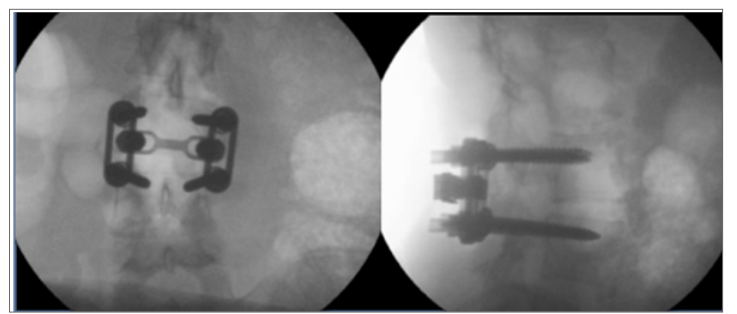

Figure 3 Plain radiograph after a L3 lamina osteoid osteoma curettage and L3-L4 posterior spine instrumentation.

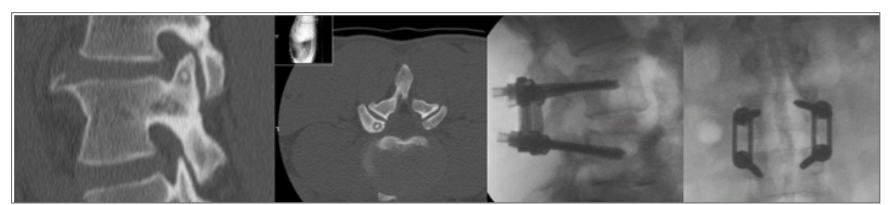

Figure $4 \mathrm{CT}$-scan of an Osteoid osteoma at L4 superior articular process and plain radiograph after L3-L4 posterior spine instrumentation.

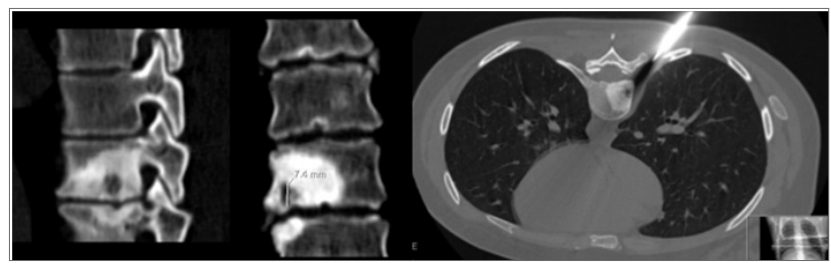

Figure 5 Osteoid osteoma at D8 vertebral body and thermoablation CTguided approach.

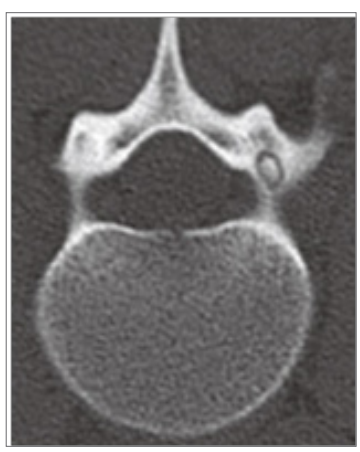

Figure 6 Osteoid osteoma at L2 pediculum.



Figure 7 Osteoid osteoma at L4 pediculum and thermoablation CT-guided approach.

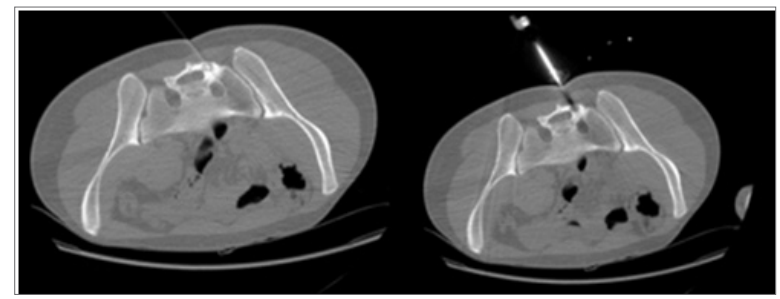

Figure 8 Osteoid osteoma at second sacral vertebra and thermoablation CT-guided approach.

\section{Discussion}

Complete resection, although historically considered the treatment of choice for $\mathrm{OO}$ with very good success rates, can present complications. To minimize surgical damage a precise location of the nidus is crucial. Conventional resection surgery is an effective option associated with low morbidity and low local recurrence rates, however RFA is emerging as an alternative method with obvious advantages. ${ }^{15,16}$ RAF is efficient, safe, less invasive, performed under local anaesthesia and allows for a faster recovery, lower costs and shorter hospital stays. Postoperative complications are similar in both methods. New protective techniques allow RFA of OO located near neurological structures with increased confidence. To safely approach tumors located near central canal or neural foramen an epidurogram can be obtained by the injection of air or carbon dioxide. The gas will outline the epidural space and increase the distance between the noble structures and the tumor. Since the heat is also a concern, techniques with the infusion of fluid at room temperature through the epidural space with a constant flow presented as a solution. Therefore, currently RFA can be used in all anatomic segments. ${ }^{17}$

Our results are according to the literature and demonstrate the safety and efficiency of both techniques.

\section{Conclusion}

Percutaneous CT-guided radiofrequency ablation of osteoid osteomas is a less invasive, time saving and economic technique with excellent long term results when compared with surgical resection.

\section{Acknowledgements}

None.

\section{Conflict of interest}

Authors declare there are no conflicts in publishing the article.

\section{References}

1. Nicolle Lindsay E. Catheter associated urinary tract infections. Antimicrob Resist Infect Control. 2014;3(1):23.

2. Amalaradjou R, Anne M, Venkitanaray K. Role of bacterial biofilms in catheter-associated urinary tract infections (CAUTI) and strategies for their control. In: Thomas N, editors. Recent advances in the field of urinary tract infections. InTech Open; 2013.

3. Laverty G, Gorman S, Gilmore BF. Biofilms and implant associated infections. In: Barnes L, Cooper Ian R, editors. Biomaterials and medical device associated infections. Cambridge, UK: Woodhead Publishers, Elsevier; 2015. p. 19-37.

4. Delcaru C, Alexandru I, Podgoreanu P, et al. Microbial biofilms in urinary tract infections and prostatitis: Etiology, pathogenicity, and combating strategies. Pathogens. 2016;5(4):E65. 
5. Wazait HD, Patel HRH, Veer V, et al. Catheter-associated urinary tract infections: prevalence of uropathogens and pattern of antimicrobial resistance in a UK Hospital (1996-2001). BJU Int. 2003;91(9):806-809.

6. Verma A, Bhani D, Tomar V, et al. Differences in bacterial colonization and biofilm formation property of uropathogens between the two most commonly used indwelling urinary catheters. $J$ Clin Diagn Res. 2016;10(6):1-3.

7. Kim B, Pai H, Choi WS, et al. Current status of indwelling urinary catheter utilization and catheter-associated urinary tract infection throughout hospital wards in Korea: A multicenter prospective observational study. PLoS One. 2017;12(10):e0185369.

8. Mihankhah A, Khoshbakht R, Raeisi M, et al. Prevalence and antibiotic resistance pattern of bacteria isolated from urinary Tract infections in Northern Iran. J Res Med Sci. 2017;22:108.

9. Singha, P, Locklin J, Handa H. A Review of the recent advances in antimicrobial coatings for urinary catheters. Acta Biomater. 2017;50:20-40

10. Holt JG. Bergey's manual of determinative bacteriology. 9 th ed. Baltimore: Willains Wilkinse; 1994. p. 1-10.
11. Clinical \& Laboratory Standards Institute. Performance standards for antimicrobial susceptibility testing twentieth. 2012;32(3):100-121.

12. Freeman DJ, Falkiner FR, Keane CT. New method for detecting slime production by coagulase negative staphylococci. J Clin Pathol. 1989;42(8):872-874.

13. O'Toole, George A. Microtiter dish biofilm formation assay. J Vis Exp. 2011;47:2437.

14. Yaakov D, Geffen Y, Andreassen S, et al. Predicting antibiotic resistance in urinary tract infection patients with prior urine cultures. Antimicrobial Agents and Chemotherapy. 2016;60(8):4717-4721.

15. Lee KH, Jung Park SU, Choi S, et al. The influence of urinary catheter materials on forming biofilms of microorganisms. Journal of Bacteriology and Virology. 2017;47(1):32-40.

16. Kumon H, Hashimoto H, Nishimura M, et al. Catheter-associated urinary tract infections: impact of catheter materials on Their management. Int $J$ Antimicrob Agents. 2001;17(4):311-316. 